\title{
ANALYTIC-NUMERICAL SOLUTIONS OF RESTRICTED NON-RESONANCE PLANAR THREE-BODY PROBLEM
}

\author{
Y. A. RYABOV \\ Moscow Automobile $\mathcal{G}$ Highway Engineering University \\ Leningradsky pr.64, Moscow, 125829, Russia
}

We consider a restricted planar circular three-body problem (Sun-Jupiter-asteroid) in a non-resonance case. There are two new algorithms developed for construction of a quasi-periodic solution in a trigonometric form by means of computer algebra. The first corresponds to classical method of simple iterations leading to series in powers of small mass $m_{J}$, the second, to iterations with rapid (quadratic) convergence, but having ordinary type and not involving a successive coordinate transformations. All these iterations require a realization of algebraic operations on trigonometric polynomials with the help of computers of high capacity. It would be interesting to compare the solutions obtained with the two algorithms and to estimate the domain of their practical convergence.

1. Let us consider the following equations of the given problem

$$
\begin{array}{rlrl}
d p / d \theta & =\mu \Phi_{1}(p, e, G, L), & & d G / d \theta=1-\mu F_{1}(p, e, G, L), \\
d e / d \theta & =\mu \Phi_{2}(p, e, G, L), & d L / d \theta=1-F_{2}(p, e, G, L),
\end{array}
$$

where $\theta$ is the longitude of asteroid, $p$ is the orbital parameter, $e$ is the eccentricity, $G$ is the true anomaly, $L$ is the difference between $\theta$ and Jupiter's longitude. $\Phi_{1}, \Phi_{2}$ are uneven and $F_{1}, F_{2}$ even functions of angular variables $G, L$. Units of mass and time are defined such that $k^{2}=1$ (gravitational constant), $m_{S}+m_{J}=1, m_{J}=\mu$ and Jupiter's semimajor axis and mean motion are $a_{J}=1$ and $n_{J}=1$. These equations and the expressions of their right-hand sides are well known. In particular, $\Phi_{1}=2 p^{3}\left[(1+e \cos G)^{-3}-\right.$ $\left.A^{-3 / 2}\right] \sin L$, where $A=p^{2}+(1+e \cos G)^{2}-2 p(1+e \cos G) \cos L$. Introducing vectors $x=(p, e), \quad y=(G, L), \quad \Phi=\left(\mu \Phi_{1}, \mu \Phi_{2}\right), \quad F\left(\mu F_{1}, F_{2}\right)$ we will seek the solution of system (1) in the form:

$$
x=x_{0}+\sum_{\|k\|=1}^{N} U_{k} \cos (k, \psi), \quad y=\psi+\sum_{\|k\|=1}^{N} V_{k} \sin (k, \psi),
$$


where $\psi=\left(\psi_{1}, \psi_{2}\right), \quad \psi_{j}=\omega_{j} \theta+\psi_{j 0}, j=1,2, \quad k=\left(k_{1}, k_{2}\right)$ is a vector with integer components $k_{1}, k_{2} . x_{0}=\left(p_{0}, e_{0}\right)$ and $\omega=\left(\omega_{1}, \omega_{2}\right)$ are vectors of the mean values $p, e$ and frequencies $\omega_{1}, \omega_{2}$, respectively; $\|k\|=$ $\left|k_{1}\right|+\left|k_{2}\right|, \quad(k, \psi)=k_{1} \psi_{1}+k_{2} \psi_{2}, \quad(k, \omega)=k_{1} \omega_{1}+k_{2} \omega_{2}$ and the number $N$ is a given maximal (sufficiently high) order of harmonics. Coefficients $U_{k}, V_{k}$ (two-dimensional vectors) and $\omega_{1}, \omega_{2}$ are unknown; $p_{0}, e_{0}$ are also unknown, but we fix their numerical values; vector $\psi_{0}=\left(\psi_{10}, \psi_{20}\right)$ is left arbitrary. We will obtain the solution (2) with numerical coefficients (i.e. with numerical components of vectors $\left.U_{k}, V_{k}, \omega\right)$ and we also fix the value of mass $\mu$. Substituting (2) into (1) we obtain the corresponding relations and our main equations in $U_{k}, V_{k}, \omega$ follows:

$$
\omega=1-\tilde{F}_{0}(U, V), \quad-(k, \omega) U_{k}=\tilde{\Phi}_{k}(U, V), \quad(k, \omega) V_{k}=-\tilde{F}_{k}(U, V),
$$

where $1 \leq\|k\| \leq N$ and $\tilde{F}_{0} \tilde{F}_{k}, \tilde{\Phi}_{k}$ are coefficients of Fourier expansions:

$$
\begin{array}{r}
\Phi(x, y)=\sum \tilde{\Phi}_{k}(U, V) \sin (k, \psi) \\
F(x, y)=\tilde{F}_{0}(U, V)+\sum \tilde{F}_{k}(U, V) \cos (k, \psi) .
\end{array}
$$

$U, V$ denote vectors whose components are all $U_{k}, V_{k}$ respectively. Coefficients $\tilde{\Phi}_{k}, \tilde{F}_{k}$ are theoretically certain expressions in different coefficients $U_{k}, V_{k}$.

2. It is essential that we can solve equations (3) by iterations in absence of analytical expressions for $\tilde{\Phi}_{k}(U, V), \tilde{F}_{k}(U, V)$.

The zero-approximation (corresponds to known formulae of unperturbed motion): $x^{0}=\left(p_{0}, e_{0}\right), \quad y^{0}=\left(G^{0}, L^{0}\right)$, where

$$
G^{0}=\omega_{1}^{0} \theta+\psi_{10}, \quad L^{0}=\omega_{2}^{0} \theta+\psi_{20}+\sum_{j=1}^{N} S_{j}^{0} \cos \left(j G^{0}\right),
$$

$\omega_{1}^{0}=1$ and $\omega_{2}^{0}, S j^{0}$ are known expressions in $p_{0}, e_{0}$; hence, $U^{0}=0, V^{0}=$ $\left(0, S_{j}^{0}\right)$. If numbers $p_{0}, e_{0}$ are given, then we obtain corresponding numbers $\omega_{2}^{0}, S_{j}^{0}$. We assume that there is no acute resonance between frequencies $\omega_{1}^{0}, \omega_{2}^{0}$.

The first approximation

$$
\begin{gathered}
\omega^{(1)}=1-\tilde{F}_{0}\left(U^{0}, V^{0}\right), \quad \psi^{(1)}=\omega^{(1)} \theta+\psi_{0}, \\
U_{k}^{(1)}=-\frac{1}{\left(k, \omega^{(1)}\right)} \tilde{\Phi}_{k}\left(U^{0}, V^{0}\right), \quad V_{k}^{(1)}=-\frac{1}{\left(k, \omega^{(1)}\right)} \tilde{F}_{k}\left(U^{0}, V^{0}\right) .
\end{gathered}
$$

It is possible to compute components of vectors $\tilde{\Phi}_{k}\left(U^{0}, V^{0}\right), \tilde{F}_{k}\left(U^{0}, V^{0}\right)$ in the following way. For example, quantities $\tilde{\Phi}_{1 k}\left(U^{0} V^{0}\right)$ are Fourier coeffcients of the function 


$$
\Phi_{1}\left(x^{0}, y^{0}\right)=2 p_{0}^{3}\left[\left(1+e_{0} \cos G^{0}\right)^{-3}-\left(A_{0}\right)^{-3 / 2}\right],
$$

where $A_{0}=p_{0}^{2}+\left(1+e_{0} \cos G^{0}\right)^{2}-2 p_{0}\left(1+e_{0} \cos G^{0}\right) \cos L^{0}, \quad G^{0}=\psi_{1}^{0}$ and $L^{0}$ is represented by (5). The algebraic manipulations of Fourier polynomials done with the help of computer algebra lead us to the expansion of form (4) with coefficients $\tilde{\Phi}_{1 k}^{0}$. Similarly, we obtain other components of mentioned vectors. Afterwards, we calculate $\omega^{(1)}, U_{k}^{(1)}, V_{k}^{(1)}$ and obtain $x^{(1)}, y^{(1)}$ in form (2) with numerical coefficients.

We can use for construction of subsequent approximations a)simple iterations and $\mathrm{b}$ )iterations with quadratic convergence.

Simple iterations $\quad \omega^{(2)}=1-\tilde{F}_{0}\left(U^{(1)}, V^{(1)}\right)$,

$$
U_{k}^{(2)}=-\frac{1}{\left(k, \omega^{(2)}\right)} \tilde{\Phi}_{k}\left(U^{(1)}, V^{(1)}\right), \quad V_{k}^{(2)}=-\frac{1}{\left(k, \omega^{(2)}\right)} \tilde{F}_{k}\left(U^{(1)}, V^{(1)}\right) .
$$

The calculations of $\tilde{F}_{k}\left(U^{(1)}, V^{(1)}\right), \tilde{\Phi}_{k}\left(U^{(1)}, V^{(1)}\right)$ are reduced to obtaining Fourier expansions of functions $F\left(x^{(1)}, y^{(1)}\right), \Phi\left(x^{(1)}, y^{(1)}\right)$, where $x^{(1)}, y^{(1)}$ are known expansions of form (2) with numerical coefficients. Subsequent approximations are defined similarly.

Iterations with quadratic convergence

In accordance with Newton's method we put in (3)

$$
\omega=\omega^{(1)}+\nu^{(1)}, \quad U_{k}=U_{k}^{(1)}+u_{k}^{(1)}, \quad V_{k}=V_{k}^{(1)}+v_{k}^{(1)}
$$

and form linearized algebraic equations in $\nu^{(1)}, u_{k}^{(1)}, v_{k}^{(1)}$ (corrections to the first approximation) introducing vectors $u=\left\{u_{k}\right\}, v=\left\{v_{k}\right\}$, whose components are sets of all $u_{k}, v_{k}$ correspondingly with $1 \leq\|k\| \leq N$. These equations are the following

$$
\begin{aligned}
\left(k, \nu^{(1)}\right) U_{k}^{(1)}+\left(k, \omega^{(1)}\right) u_{k}^{(1)}+\left(\partial \tilde{\Phi}_{k} / \partial U\right)_{1} u^{(1)}+\left(\partial \tilde{\Phi}_{k} / \partial V\right)_{1} v^{(1)} & =\Delta \tilde{\Phi}_{(k)}^{(1)} \\
\left(k, \nu^{(1)}\right) V_{k}^{(1)}+\left(k, \omega^{(1)}\right) v_{k}^{(1)}+\left(\partial \tilde{F}_{k} / \partial U\right)_{1} u^{(1)}+\left(\partial \tilde{F}_{k} / \partial V\right)_{1} v^{(1)} & =\Delta \tilde{F}_{k}^{(1)} \\
\nu^{(1)}+\left(\partial \tilde{F}_{0} / \partial U\right)_{1} u^{(1)}+\left(\partial \tilde{F}_{0} / \partial V\right)_{1} v^{(1)} & =\Delta \tilde{F}_{0}^{(1)}
\end{aligned}
$$

where $1 \leq\|k\| \leq N, \Delta \tilde{\Phi}_{k}^{(1)}=\tilde{\Phi}_{k}^{0}-\tilde{\Phi}_{k}^{(1)}$ etc. and $\tilde{\Phi}_{k}^{(j)}=\tilde{\Phi}_{k}\left(U^{(j)}, V^{(j)}\right)$ etc. The derivative $\left(\partial \tilde{\Phi}_{k} / \partial U\right)_{1}=\partial \tilde{\Phi}_{k} /\left.\partial U\right|_{U=U^{(1)}, V=V^{(1)}}$ is the block matrix $\left[C_{l 1, l 2}\right]$ with $C_{l 1, l 2}$ being $2 \times 2-$ matrices $\left(\partial \tilde{\Phi}_{k} / \partial U_{l 1, l 2}\right)_{1}$.

According to the expansion of $\Phi\left(x^{(1)}, y^{(1)}\right)$ we have

$$
\Phi\left(x^{(1)}, y^{(1)}\right)=\sum_{\|k\|=1}^{N} \tilde{\Phi}_{k}\left(U^{(1)}, V^{(1)}\right) \sin (k, \psi),
$$


and for fixed vector $l=(l 1, l 2)$

$$
\frac{\partial \Phi}{\partial U_{l 1, l 2}}=\frac{\partial \Phi}{\partial x} \cos (l, \psi)=\sum_{\|k\|=1}^{N} \frac{\partial \tilde{\Phi}_{k}}{\partial U_{l 1, l 2}} \sin (k, \psi) .
$$

Hence, $\left(\partial \tilde{\Phi}_{k} / \partial U_{l 1, l 2}\right)_{1}$ for different vectors $k$ are Fourier matrix-coefficients for the function $\Phi^{\star}=(\partial \Phi / \partial x)_{1} \cos (l, \psi)$.

We obtain Fourier expansion for this function by means of manipulations considered above. Others derivatives are calculated similarly. Certainly, these calculations are very cumbersome, but they are feasible by using computers of sufficient capacity.

Having calculated $\nu^{(1)}, u_{k}^{(1)}, v_{k}^{(1)}$, we obtain the second approximation for $U_{k}, V_{k}, \omega$ and the second approximation for $x, y$ in form (2).

Algebraic equations for the corrections $u_{k}^{(2)}, v_{k}^{(2)}, \nu^{(2)}$ are formed in the similar way. The left-hand sides of these equations differ from the left-hand sides of equations in $u^{(1)}, v^{(1)}, \nu^{(1)}$ only in their super- or subscripts: (2) instead (1); on the right-hand sides we obtain the following functions:

$$
\begin{gathered}
\tilde{\Phi}_{k}\left(U^{(2)}, V^{(2)}\right)-\tilde{\Phi}_{k}\left(U^{(1)}, V^{(1)}\right)-\left(\partial \tilde{\Phi}_{k} / \partial U\right)_{1} u^{(1)}-\left(\partial \tilde{\Phi}_{k} / \partial V\right)_{1} v^{(1)} \\
\left.\left.\tilde{F}_{k}\left(U^{(2)}, V(2)\right)-\tilde{F}_{k}\left(U^{(1)}, V^{(1)}\right)-\left(\partial \tilde{F}_{k} / \partial U\right)\right)_{1} u^{(1)}-\left(\partial \tilde{F}_{k} / \partial V\right)\right)_{1} v^{(1)}
\end{gathered}
$$

We obtain, after calculation of $u_{k}^{(2)}, v_{k}^{(2)}, \nu_{k}^{(2)}$, the third approximation, etc. The computations are ended when differences between two adjacent approximations for $\omega$ and for all $U_{k}, V_{k}$ are less, in norm, than a given quantity $\delta$. These iterations possess quadratic type of convergence in relation to small mass $\mu$ if we leave out of account possible small divisors $\left(k, \omega^{(j)}\right)$. Taking into consideration results of KAM-theory we could hope that quadratic convergence will compensate above mentioned small divisors in absence of an acute resonance between $\omega_{1}^{0}, \omega_{2}^{0}$.

Certainly, it arises the question about practical effectiveness of proposed algorithms in the course of immediate computations. If results are positive, the algorithm may be complicated for the purpose of considering 3-dimensional three-body problem and also to leave arbitrary some quantities as, for example, the mass $\mu$.

\section{References}

Grebenikov E.,Ryabov Y. Constructive methods in the analysis of nonlinear systems.Moscow, ed.MIR,1983, 324 p. 